\title{
Chapter 13 \\ Is there a Role for Neoadjuvant Targeted Therapy and Immunotherapy?
}

\author{
Simon Beyaert and Jean-Pascal Machiels
}

Abbreviations

${ }^{18}$ FDG-PET 18- fluorodeoxyglucose-positron emission tomography

EGFR Epidermal growth factor receptor

G-CSF Granulocyte-colony stimulating factor

$\mathrm{PD}(-\mathrm{L}) \quad$ Programmed death (-Ligand)

SAE Severe adverse events

SCCHN Squamous cell carcinoma of the head and neck

SUV Standardized uptake value

TPF Taxanes, platinum-based chemotherapy and 5-fluorouracil

\section{Introduction}

The role of induction or neoadjuvant therapy to treat locally advanced squamous cell carcinoma of the head and neck (SCCHN) is controversial [1, 2]. Standard treatment remains concomitant chemoradiation with high-dose $\left(100 \mathrm{mg} / \mathrm{m}^{2}\right)$ cisplatin when a non-surgical approach is preferred $[1,2]$. The only recognized indication for induction chemotherapy is larynx preservation, and the oncological outcome is similar to that of concomitant chemoradiation in this particular setting [3]. Taxane/ platinum/5-Fluorouracil (TPF) combinations have proven to be superior to

\footnotetext{
S. Beyaert

Institut de Recherche Expérimentale et Clinique (Pôle MIRO), Université Catholique de Louvain (UCLouvain), Brussels, Belgium

e-mail: simon.beyaert@uclouvain.be

J.-P. Machiels $(\bowtie)$

Institut de Recherche Expérimentale et Clinique (Pôle MIRO), Université Catholique de Louvain (UCLouvain), Brussels, Belgium

Service d'Oncologie Médicale, Institut Roi Albert II, Cliniques universitaires Saint-Luc, Brussels, Belgium

e-mail: jean-pascal.machiels@uclouvain.be
} 
platinum/5-fluorouracil schedules, and TPF is therefore now the accepted standard for induction $[4,5]$.

In this chapter, we review if there is a role forneoadjuvant targeted therapy or immunotherapy in the treatment of SCCHN. We discuss two different approaches: neoadjuvant or induction studies and window of opportunity trials.

\section{Induction Therapy with Targeted Therapy and Immune Checkpoint Inhibitors}

In a neoadjuvant or induction approach, the definitive standard treatment (i.e. surgery or (chemo)radiation) is delayed to allow enough time for the neoadjuvant agent(s) to produce a therapeutic response and improve overall treatment efficacy. In this setting, the use of drugs targeting the epidermal growth factor receptor (EGFR) has been largely investigated in combination with chemotherapy.

Three trials have studied the safety and feasibility of combining cetuximab with TPF [6-8]. Specenier et al. investigated four cycles of TPF plus cetuximab (TPF-E) (cisplatin and docetaxel $75 \mathrm{mg} / \mathrm{m}^{2}$ on day 1 followed by 5 -fluoruracil (5-FU) $750 \mathrm{mg} / \mathrm{m}^{2} /$ day as a continuous infusion on days $1-5$ plus cetuximab at a loading dose of $400 \mathrm{mg} / \mathrm{m}^{2}$ followed by a weekly dose of $250 \mathrm{mg} / \mathrm{m}^{2}$ ), with prophylactic antibiotics but no growth factors [6]. Induction TPF-E was discontinued in 13\% of patients due to toxicity, and three out of 46 patients developed a bowel perforation. Only $65 \%$ of the patients in this study started chemoradiation. Mesia et al., using the same TPF regimen but with prophylactic granulocyte-colony stimulating factor (G-CSF) and antibiotics, observed febrile neutropenia, grade III/IV diarrhea and toxic death in $24 \%, 20 \%$ and $6 \%$ of patients, respectively [7]. It was therefore deemed that TPF-E leads to unacceptable toxicities. In contrast, Haddad et al. found that it was feasible to give three cycles of TPF-E with cisplatin $100 \mathrm{mg} / \mathrm{m}^{2}$ day 1 , docetaxel $75 \mathrm{mg} / \mathrm{m}^{2}$ day 1 and $5-\mathrm{FU} 850 \mathrm{mg} / \mathrm{m}^{2} /$ day as a continuous infusion on days 1-4 plus cetuximab for a total of six weeks given on days 1 and 8 of each cycle of TPF [8]. Similarly, a phase I trial combined lapatinib with TPF, but this combination also resulted in prohibitive toxicities [9].

Therefore, several single arm phase II trials evaluated the combination of cetuximab with a platinum compound and a taxane but without 5-FU [10-14]. In most of the trials, these combinations were found to be feasible, and observed objective response rates of between $70 \%$ and $97 \%$ were promising (Table 13.1).

A small number of randomized trials have compared cetuximab/platinum/ taxane-based induction chemotherapy versus $\mathrm{TP}(\mathrm{F})$ [15-17]. No clinically significant differences were observed between the cetuximab-based regimens and the controls (Table 13.2). Therefore, the role of induction therapy with a targeted agent to treat SCCHN remains purely investigational. 
Table 13.1 Single arm phase II trials investigating cetuximab with a platinum compound and a taxane

\begin{tabular}{l|l|l|l|l}
\hline Regimens & N & ORR & $\begin{array}{l}3 \text {-year PFS } \\
\text { rate }\end{array}$ & $\begin{array}{l}3 \text {-year } \\
\text { OS }\end{array}$ \\
\hline Cisplatin + Docetaxel + Cetuximab [10] & 39 & $86 \%$ & $70 \%$ & $74 \%$ \\
\hline Cisplatin + Docetaxel + Cetuximab [11] & 54 & $72.2 \%$ & $58.2 \%$ & $90.7 \%$ \\
\hline $\begin{array}{l}\text { Carboplatin (AUC2) + Paclitaxel (135 mg/m2/ } \\
\text { weeks) + Cetuximab [12] }\end{array}$ & 47 & $96 \%$ & $87 \%$ & $91 \%$ \\
\hline $\begin{array}{l}\text { Carboplatin (AUC2) + Paclitaxel (90mg/m2/weeks) + } \\
\text { Cetuximab [13] }\end{array}$ & 30 & $97 \%$ & NA & NA \\
\hline $\begin{array}{l}\text { Carboplatin (AUC2) + Paclitaxel (90 mg/m2/ } \\
\text { weeks) + Cetuximab [14] }\end{array}$ & 63 & $70 \%$ & $55 \%$ & $78 \%$ \\
\hline
\end{tabular}

$N A$ not-available; $A U C$ area under the curve: $O R R$ objective response rate; $P F S$ progression-free survival; $O S$ overall survival; $y$ year

Table 13.2 Randomized phase II trials investigating cetuximab with a platinum compound and a taxane

\begin{tabular}{l|l|l|l|l}
\hline Regimens & $\mathrm{N}$ & ORR & 3-year PFS rate & 3-year OS \\
\hline Cisplatin + Docetaxel & 44 & $82 \%$ & $56 \%$ & $74 \%$ \\
\hline Versus & & & & \\
\hline Cisplatin + Docetaxel + Cetuximab [15] & 48 & $81 \%$ & $70 \%$ & $88 \%$ \\
\hline Regimens & $\mathrm{N}$ & ORR & 400 -day PFS rate & $\begin{array}{l}400 \text {-day OS } \\
\text { rate }\end{array}$ \\
\hline Cisplatin + Docetaxel +5-fluorouracil & 50 & $77 \%$ & $67 \%$ & $86 \%$ \\
\hline Versus & & & & $79 \%$ \\
\hline Cisplatin + Docetaxel + Cetuximab [16] & 50 & $86 \%$ & $70 \%$ & 2 -year OS rate \\
\hline Regimens & $\mathrm{N}$ & ORR & 2 -year LFS rate & $68 \%$ \\
\hline Cisplatin + Docetaxel + (5-fluorouracil) & 180 & $82 \%$ & $46 \%$ & $69 \%$ \\
\hline Versus & & & & \\
\hline $\begin{array}{l}\text { Cisplatin + Docetaxel + } \\
\text { (5-Fluorouracil) + Cetuximab [17] }\end{array}$ & & $81 \%$ & $47 \%$ & \\
\hline
\end{tabular}

$N A$ not-available; $A U C$ area under the curve: $O R R$ objective response rate; $P F S$ progression-free survival; $L F S$ laryngectomy-free survival: $O S$ overall survival; $y$ :year

Based on the promising efficacy of some window trials, immune checkpoint inhibitors are also under evaluation. A phase III trial is currently investigating the standard of care versus two cycles of neoadjuvant pembrolizumab (200 mg every 3 weeks) followed by curative-intent surgery and postoperative pembrolizumabbased (chemo)radiation [18]. One of the primary endpoints is pathological response after neoadjuvant therapy $(<10 \%$ of tumor cells within the resected primary tumor and lymph nodes). The study design is depicted in Fig. 13.1. 


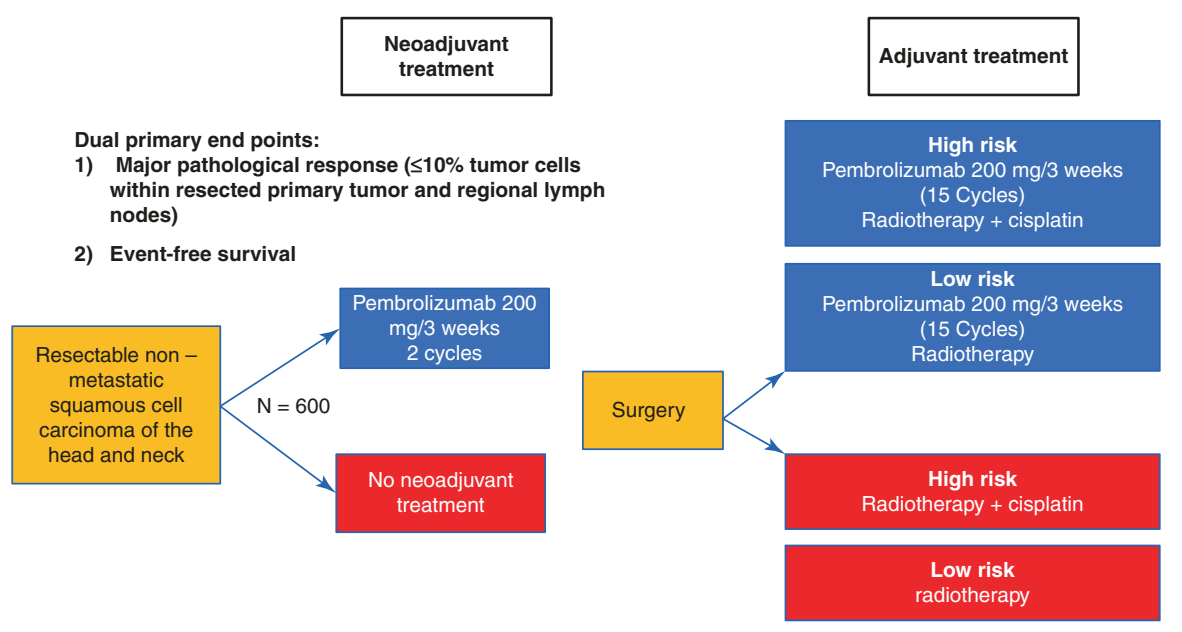

Fig. 13.1 KEYNOTE-689: Phase III study of adjuvant and neoadjuvant pembrolizumab combined with standard of care in patients with resectable, locally advanced head and neck squamous cell carcinoma

\section{Window of Opportunity Trials with Targeted Therapy and Immune Checkpoint Inhibitors}

Window of opportunity studies are trials in which patients receive one new compound in the period between their cancer diagnosis and the start of standard therapy. The primary objective of this approach is not treatment efficacy but translational research. Standard treatment is usually surgery. Tumor biopsies and anatomic and functional imaging are performed before and after investigational treatment for translational research (Fig. 13.2). The main advantage of this study design is the ability to investigate new molecules in patients who have not yet been treated by multiple anti-cancer therapies. Traditionally, drugs are often tested in patients with locoregional or metastatic recurrence whose tumors are predominantly resistant and there is a risk that the activity of these agents may be underestimated. Furthermore, the understanding of the biological and molecular effects of these tested drugs may be limited in palliative patients because it could be perceived unethical to perform additional biopsies for research purposes only. In head and neck cancer window studies, pretreatment biopsies during diagnostic endoscopy and post-treatment biopsies on the day of surgery can be performed, taking advantage of general anesthesia. The evaluation of new compounds using this approach prior to classical curative treatment provides information about molecular and clinical activity as well as predictive biomarkers [19, 20].

Window of opportunity studies aim to maximize the information gain whilst minimizing the risk to patients who are awaiting potentially curative treatment. Standard treatment should therefore not be delayed due to the investigational drugs' study procedures or side effects. Some studies have shown that curative treatment in head and neck cancer should be carried out within 20 to 28 days after diagnosis $[21,22]$, 


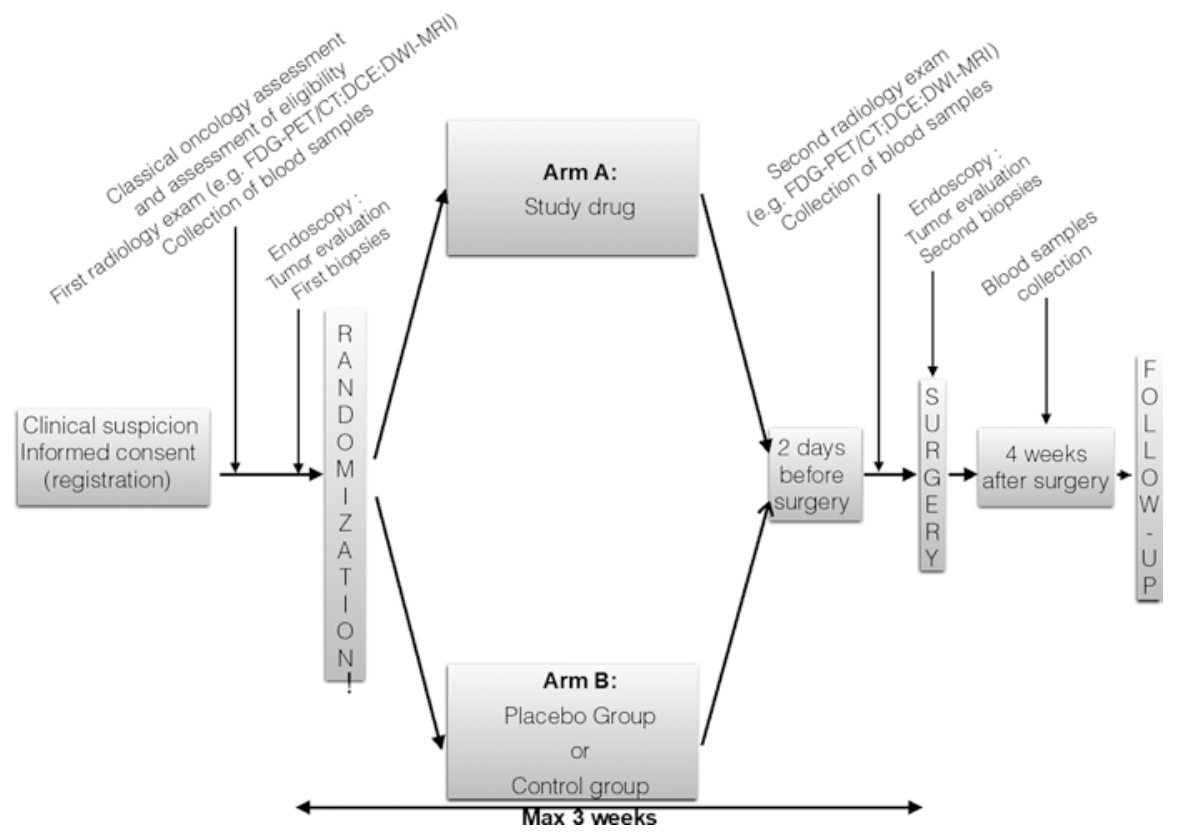

Fig. 13.2 Example of a window of opportunity study design. FDG-PET fluorodeoxyglucosepositron emission tomography; DCE dynamic contrast enhanced; DWI-MRI diffusion-weighted magnetic resonance imaging

making trial organization one of the main challenges for this type of study. To achieve this goal, we recommend that patients with SCCHN be included in window studies at the time of clinical diagnosis, that the time points for biopsies and imaging are prospectively pre-defined, and that the schedule, dose, and duration of the preoperative treatment are standardized and the same for all patients. Finally, to validate translational research, patients should also be randomized against a control/ placebo group. If macroscopic tumor reduction is observed with the investigational compound, surgery should be performed as initially planned to ensure that the extracted surgical specimen has clear margins without microscopic tumor invasion.

Several PD1/PD-L1 monoclonal antibodies and anti-EGFR agents have been investigated in this setting (Tables 13.3 and 13.4). Interestingly, and aside from molecular activity, clinical efficacy has sometimes been detected even if the treatment period was short ( $<4$ weeks). We will discuss some examples to highlight the advantages and drawbacks of this research approach.

Schmitz et al. [21, 23, 24] investigated cetuximab versus controls in the two weeks before curative surgery in treatment-naïve patients with SCCHN. The primary endpoint of safety was reached with cetuximab prior to surgery. Cetuximab also induced a high rate of response based on 18-fluorodeoxyglucose-positron emission tomography $\left({ }^{18} \mathrm{FDG}-\mathrm{PET}\right)$ evaluation and a decrease in tumor cellularity, which significantly correlated with ${ }^{18}$ FDG-PET response. Four patients out of 20 also had a 


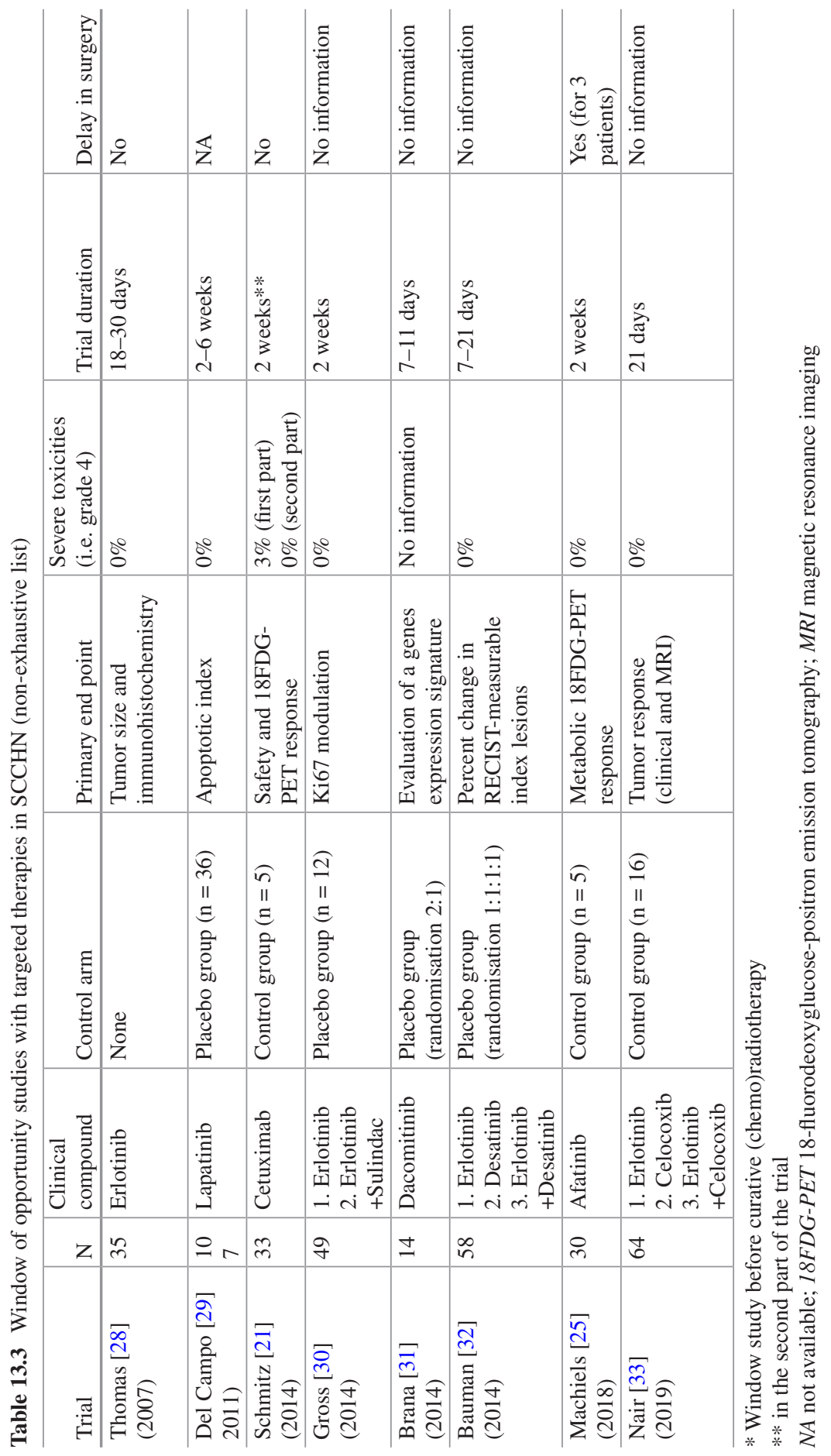




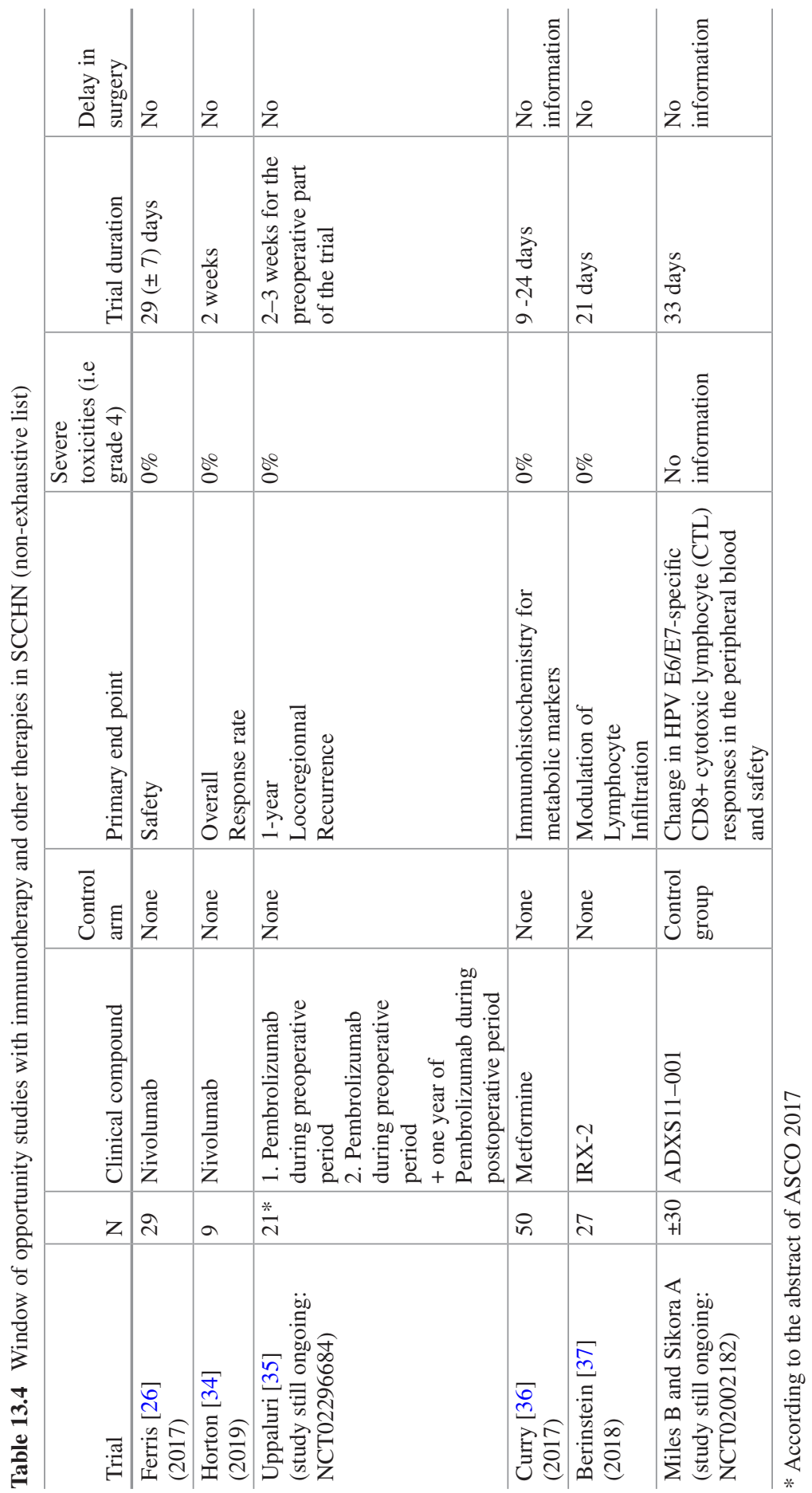


macroscopic reduction in the size of their tumor. Gene expression analyses showed that in some patients cetuximab increased the expression of genes involved in epithelial to mesenchymal transition and activation of cancer-associated fibroblasts.

Afatinib, an irreversible pan-ErbB inhibitor, has also been investigated in a multicenter randomized window study of 25 treated patients versus five controls [25]. The primary endpoint was ${ }^{18}$ FDG-PET response. Seventy percent of the patients showed a partial metabolic response and $22 \%$ of patients had a partial response according to RECIST v1.1. A high cluster 3-hypoxia score and wild TP53 status were predictive of treatment activity. The investigational compound was considered safe even though three patients experienced surgical delay. Among them, two delays ( 3 and 24 days, respectively) were related to drug toxicity. We therefore believe that it is preferable to use drugs that have already proven to be safe in phase I studies in order to maximize patient safety and to protect the initiation of standard treatment. To the best of our knowledge, very few window studies in head and neck oncology have had to deal with grade $\geq 4$ or unexpected side effects.

In 2017, Ferris et al. [26] conducted a window study with nivolumab, a monoclonal antibody targeting PD-1, in 29 SCCHN patients. Patients received two doses prior to surgery that was planned on day $29 \pm 7$. The primary endpoint was safety. The publication is still pending, but according to the ESMO 2017 abstract, grade 3-4 treatment-related adverse events occurred in four patients without delaying surgery. Tumor shrinkage, assessed by computer tomography (CT)-scan just before surgery, was observed in $48 \%$ of evaluable patients. Three patients experienced tumor reduction $\geq 40 \%$ (largest reduction $=75 \%$ ). However, 11 patients also showed an increase in tumor size (the largest by 100\%). At this stage, it is not possible to differentiate between true tumor progression or pseudo-progression.

More recently, vaccine-based therapies have begun to be investigated using window study designs. The main challenge for vaccines using this trial design is the limited period of time that short-term vaccination has available to show effective immunological effects. In this context, we recommend the use of minimally invasive samples (e.g. blood tests) to investigate the therapeutic effect of these vaccines after standard curative treatment, for example four weeks after surgery, as shown in Fig. 13.2. A metaanalysis of 239 phase I therapeutic cancer vaccine trials, conducted by Rahma et al. [27], concluded that the risk of severe adverse events (SAEs) when testing therapeutic cancer vaccines is extremely low and that AEs did not correlate with dose levels. Several window studies investigating the use of short-term therapeutic vaccination in head and neck cancers are currently in progress. First results are pending.

\section{Conclusion}

Targeted and immune therapies as induction or neoadjuvant therapy are not standard of care and should be reserved for clinical trials. In this context, a phase III trial is investigating neoadjuvant and adjuvant pembrolizumab in patients selected for a primary surgical treatment. Window of opportunity trials are important translational research tools that require careful design and an experienced team. 


\section{References}

1. Blanchard P, Baujat B, Holostenco V, et al. Meta-analysis of chemotherapy in head and neck cancer (MACH-NC): a comprehensive analysis by tumour site. Radiother Oncol. 2011;100(1):33-40.

2. Pignon JP, le Maître A. Bourhis J; MACH-NC collaborative group. Meta-analyses of chemotherapy in head and neck cancer (MACH-NC): an update. Int J Radiat Oncol Biol Phys. 2007;69(2 Suppl):S112-4.

3. Forastiere AA, Ismaila N, Lewin JS, et al. Use of larynx-preservation strategies in the treatment of laryngeal cancer: American Society of Clinical Oncology clinical practice guideline update. J Clin Oncol. 2018;36(11):1143-69.

4. Vermorken JB, Remenar E, van Herpen C, et al. Cisplatin, fluorouracil, and docetaxel in unresectable head and neck cancer. N Engl J Med. 2007;357(17):1695-704.

5. Posner MR, Hershock DM, Blajman CR, et al. Cisplatin and fluorouracil alone or with docetaxel in head and neck cancer. N Engl J Med. 2007;357(17):1705-15.

6. Specenier PM, Remenar E, Buter J, et al. TPF plus cetuximab induction chemotherapy followed by biochemoradiation with weekly cetuximab plus weekly cisplatin or carboplatin: a randomized phase II EORTC trial. Ann Oncol. 2017;28(9):2219-24.

7. Mesía R, Vázquez S, Grau JJ, et al. A phase 2 open label, single-arm trial to evaluate the combination of cetuximab plus taxotere, cisplatin, and 5-flurouracil as an induction regimen in patients with unresectable squamous cell carcinoma of the head and neck. Int J Radiat Oncol Biol Phys. 2016;94(2):289-96.

8. Haddad RI, Tishler RB, Norris C, et al. Phase I study of C-TPF in patients with locally advanced squamous cell carcinoma of the head and neck. J Clin Oncol. 2009;27(27):4448-53.

9. Lalami Y, Specenier PM, Awada A, et al. EORTC 24051: unexpected side effects in a phase I study of TPF induction chemotherapy followed by chemoradiation with lapatinib, a dual EGFR/ErbB2 inhibitor, in patients with locally advanced resectable larynx and hypopharynx squamous cell carcinoma. Radiother Oncol. 2012;105(2):238-40.

10. Argiris A, Heron DE, Smith RP, et al. Induction docetaxel, cisplatin, and cetuximab followed by concurrent radiotherapy, cisplatin, and cetuximab and maintenance cetuximab in patients with locally advanced head and neck cancer. J Clin Oncol. 2010;28(36):5294-300.

11. Zenda S, Ota Y, Kiyota N, et al. A multicenter phase II trial of docetaxel, cisplatin, and cetuximab (TPEx) followed by cetuximab and concurrent radiotherapy for patients with local advanced squamous cell carcinoma of the head and neck (CSPOR HN01: ECRIPS study). Front Oncol. 2019;9:6. Published 2019 Jan 22

12. Kies MS, Holsinger FC, Lee JJ, et al. Induction chemotherapy and cetuximab for locally advanced squamous cell carcinoma of the head and neck: results from a phase II prospective trial. J Clin Oncol. 2010;28(1):8-14.

13. Bauman J, Langer C, Quon H, et al. Induction chemotherapy with cetuximab, carboplatin and paclitaxel for the treatment of locally advanced squamous cell carcinoma of the head and neck. Exp Ther Med. 2013;5(4):1247-53.

14. Wanebo HJ, Lee J, Burtness BA, et al. Induction cetuximab, paclitaxel, and carboplatin followed by chemoradiation with cetuximab, paclitaxel, and carboplatin for stage III/IV head and neck squamous cancer: a phase II ECOG-ACRIN trial (E2303). Ann Oncol. 2014;25(10):2036-41.

15. Lee KW, Koh Y, Kim SB, et al. A randomized, multicenter, phase II study of cetuximab with docetaxel and cisplatin as induction chemotherapy in unresectable, locally advanced head and neck cancer. Oncologist. 2015;20(10):1119-20.

16. Haddad RI, Massarelli E, Lee JJ, et al. Weekly paclitaxel, carboplatin, cetuximab, and cetuximab, docetaxel, cisplatin, and fluorouracil, followed by local therapy in previously untreated, locally advanced head and neck squamous cell carcinoma. Ann Oncol. 2019;30(3):471-7.

17. Dietz A, Wichmann G, Kuhnt T, et al. Induction chemotherapy (IC) followed by radiotherapy (RT) versus cetuximab plus IC and RT in advanced laryngeal/hypopharyngeal cancer resectable only by total laryngectomy-final results of the larynx organ preservation trial DeLOS-II. Ann Oncol. 2018;29(10):2105-14. 
18. Uppaluri R, Lee N, Westra W et al. KEYNOTE-689: phase 3 study of adjuvant and neoadjuvant pembrolizumab combined with standard of care (SOC) in patients with resectable, locally advanced head and neck squamous cell carcinoma. J Clin Oncol 2019; TPS6090 (abstr).

19. Schmitz S, Duhoux F, Machiels JP. Window of opportunity studies: do they fulfil our expectations? Cancer Treat Rev. 2016;43:50-7.

20. Zandberg DP, Ferris RL. Window studies in squamous cell carcinoma of the head and neck: values and limits. Curr Treat Options in Oncol. 2018;19(12):68.

21. Schmitz S, Hamoir M, Reychler H, et al. Tumour response and safety of cetuximab in a window pre-operative study in patients with squamous cell carcinoma of the head and neck. Ann Oncol. 2013;24(9):2261-6.

22. Primdahl H, Nielsen AL, Larsen S, et al. Changes from 1992 to 2002 in the pretreatment delay for patients with squamous cell carcinoma of larynx or pharynx: a Danish nationwide survey from DAHANABOUT. Acta Oncol. 2006;45:156-61.

23. Schmitz S, Rommel D, Michoux N, et al. Dynamic contrast-enhanced computed tomography to assess early activity of cetuximab in squamous cell carcinoma of the head and neck. Radiol Oncol. 2015;49(1):17-25.

24. Schmitz S, Bindea G, Albu RI, Mlecnik B, Machiels JP. Cetuximab promotes epithelial to mesenchymal transition and cancer associated fibroblasts in patients with head and neck cancer. Oncotarget. 2015;6(33):34288-99.

25. Machiels JP, Bossi P, Menis J, Lia M, Fortpied C, Liu Y, et al. Activity and safety of afatinib in a window preoperative EORTC study in patients with squamous cell carcinoma of the head and neck (SCCHN). Ann Oncol. 2018;29(4):985-91.

26. Ferris RL, Goncalves A, Baxi S, Martins UM, Gauthier H, Langenberg M, et al. LBA46-an open-label, multicohort, phase $1 / 2$ study in patients with virus- associated cancers (CheckMate 358): safety and efficacy of neoadjuvant nivolumab in squamous cell carcinoma of the head and neck. Ann Oncol. 2017;28(suppl_5):ESMO 2017 Congress.

27. Rahma OE, Gammoh E, Simon RM, Khleif SN. Is the "3+3" dose-escalation phase I clinical trial design suitable for therapeutic cancer vaccine development? A recommendation for alternative design. Clin Cancer Res. 2014;20(18):4758-67.

28. Thomas F, Rochaix P, Benlyazid A, et al. Pilot study of neoadjuvant treatment with erlotinib in nonmetastatic head and neck squamous cell carcinoma. Clin Cancer Res. 2007;13(23):7086-92.

29. Del Campo JM, Hitt R, Sebastian P, et al. Effects of lapatinib monotherapy: results of a randomised phase II study in therapy-naive patients with locally advanced squamous cell carcinoma of the head and neck. Br J Cancer. 2011;105(5):618-27.

30. Gross ND, Bauman JE, Gooding WE, et al. Erlotinib, erlotinib-sulindac versus placebo: a randomized, double-blind, placebo-controlled window trial in operable head and neck cancer. Clin Cancer Res. 2014;20(12):3289-98.

31. Brana I, She D, Chau NG, Pham N-A, Kim L, Sakashita S, et al. Preoperative window-ofopportunity (WOO) study of dacomitinib (Dac) in patients (Pts) with resectable oral cavity squamous cell carcinoma (OCC): generation of a gene signature (DGS) as a predictor of Dac activity. J Clin Oncol. 2014;32(151):6041.

32. Bauman JE, Duvvuri U, Gooding WE, et al. Erlotinib, dasatinib, erlotinib-dasatinib versus placebo: a randomized, double-blind window study in operable head and neck squamous cell carcinoma (HNSCC). J Clin Oncol. 2014;32(151):6033.

33. Nair SV, Joshi A, Patil VM, et al. A phase II randomized control trial of erlotinib in combination with celecoxib in patients with operable oral squamous cell carcinoma (OSCC): Erlo-Xib study. J Clin Oncol. 2019;37(15):6054.

34. Horton JD, Knochelmann H, Armeson K, et al. Neoadjuvant presurgical PD-1 inhibition in oral cavity squamous cell carcinoma. J Clin Oncol. 2019;37(15):2574.

35. Uppaluri R, Zolkind P, Lin T, Nussenbaum B, Jackson R, Rich J, et al. Neoadjuvant pembrolizumab in surgically resectable, HPV negative, locally advanced head and neck squamous cell carcinoma (HNSCC). J Clin Oncol. 2017;35(151):6012. 
36. Curry J, Johnson J, Tassone P, et al. Metformin effects on head and neck squamous carcinoma microenvironment: window of opportunity trial. Laryngoscope. 2017;127(8):1808-15.

37. Berinstein NL, McNamara M, Nguyen A, Egan J, Wolf GT. Increased immune infiltration and chemokine re- ceptor expression in head and neck epithelial tumors after neoadjuvant immunotherapy with the IRX-2 reg- imen. Onco Targets Ther. 2018;7(5):e1423173.

Open Access This chapter is licensed under the terms of the Creative Commons Attribution 4.0 International License (http://creativecommons.org/licenses/by/4.0/), which permits use, sharing, adaptation, distribution and reproduction in any medium or format, as long as you give appropriate credit to the original author(s) and the source, provide a link to the Creative Commons license and indicate if changes were made.

The images or other third party material in this chapter are included in the chapter's Creative Commons license, unless indicated otherwise in a credit line to the material. If material is not included in the chapter's Creative Commons license and your intended use is not permitted by statutory regulation or exceeds the permitted use, you will need to obtain permission directly from the copyright holder. 